\title{
Do Oil and Gas Revenues Promote Economic Diversification in Qatar?
}

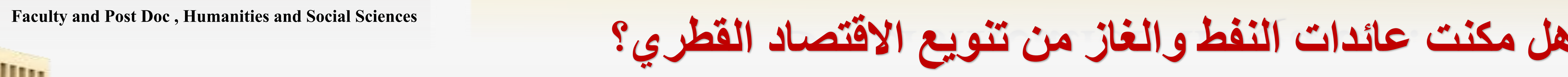

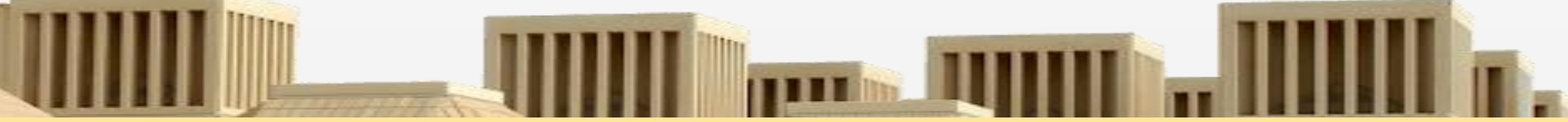

Dr. Lanouar Charfeddine

Dr. Karim Barkat

\section{ntroduction}

The state of Qatar has made the development of a competitive and diversified economy one of its top priorities. The first pillar of its National Vision 2030, the economic development pillar, is intended to optimize the use and the sustainability of Qatar's natural resources (oil and gas revenues).

Recognizing that the reliance on the abundant natural resources left the country vulnerable to the dynamic fluctuations of world energy prices, the Qatari government has massively invested its accumulated oil and gas revenues in the non-oil activities by prioritizing investments in different sectors such as tourism, sport, transport and infrastructure.

A recurrent question that arises is whether these investments have significantly contributed to diversify the economy of Qatar and delink its government revenues from the oil and gas sector.

\section{Goal and objectives}

Goal: The aim of this paper is to investigate to what extent oil and gas revenues have contributed to Qatar economic diversification.

$\square$ Objectives: Precisely we will answer the following questions:

- Do oil and gas revenues contribute to Qatar economic diversification? - Does the Qatar economy resilient to negative oil prices shocks?

- Does the Resource curse hypothesis hold for the economy of Qatar?

\section{Materials \& Methods}

A. Data sources and variables definitions

Period: Quarterly data spanning from 2000Q1 to 2018Q1 ( $T=75$ observations).

\section{Variables definitions:}

- Real oil and gas revenues (ROGR) is the total oil and gas revenues (constant US dollar 2004)

Non-oil real GDP (RNGDP) is the non-oil real gross domestic product (constant US dollar 2004) is proxy of Economic diversification.

Real exchange rate $(R E R)$ is defined such that an increase means a real depreciation of the Qatari Riyal.

- Inflation rate is the quarterly changes in the Qatari consumer prices.

Data source: All data are collected from the central bank of Qatar and the International Energy Agency.

Dummy variables: DU03 (Iraq-Us War, 2003:I-2003:IV), DU05 (Liquefied Gas, 2005:II-2006:I), DU08 (Global Financial Crisis, 2007:IV2010:IV), DU14 (GCC crisis, 2014:II-2014:IV)

15
14
14.4
13
1
128
12.4
1

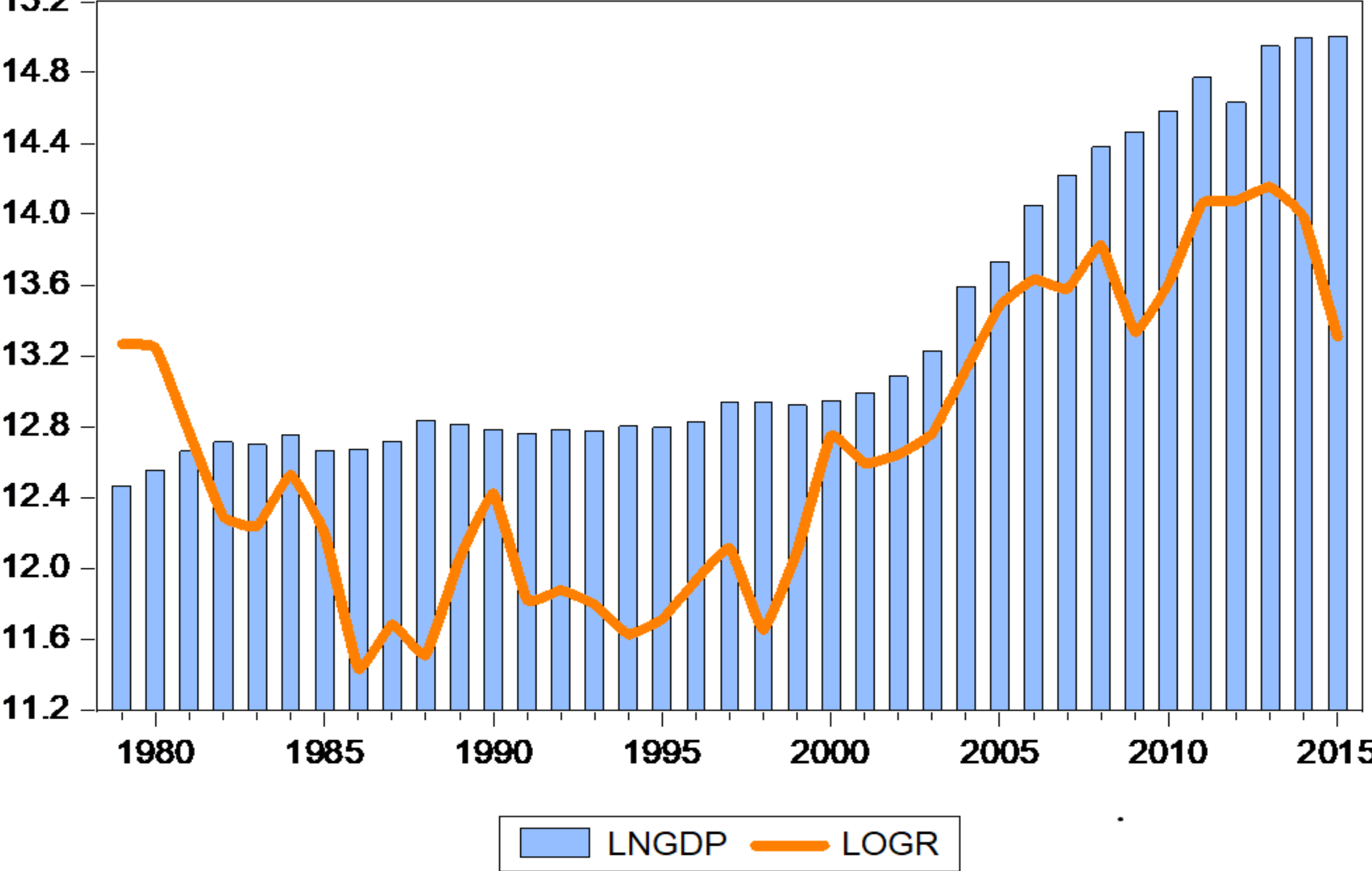

Figure 1: Real oil and gas revenues and real non-oil GDP in Qatar

\section{B. Econometric Models}

conometric approaches have been used:

variables.

The nonlinear autoregressive distributed lag (NARDL) model.

B.1. The $A-B-S V A R X$ model

$A Y_{t}=A_{1} Y_{t-1}+A_{2} Y_{t-2}+\cdots+A_{p} Y_{t-p}+C X_{t}+B \quad u_{t}$

Where, $Y_{t}$ represents the $(n \times 1)$ vector of the vait
$Y_{t}=\left[R O G R^{+} R O G R_{-}^{-}, R E R_{t} I N F_{t} R N G D P_{t}\right]$

The relationship between the reduced form innovations $\left(\varepsilon_{t}\right)$ and the structural shocks

$\left(u_{t}\right)$ is given by: $A \varepsilon_{t}=B u_{t}$

The model take the matrix form,

$\left[\begin{array}{lllll}1 & 0 & 0 & 0 & 0 \\ X & 1 & 0 & 0 & 0 \\ X & X & 1 & 0 & 0 \\ X & X & X & 1 & 0 \\ X & X & X & X & 1\end{array}\right]\left[\begin{array}{c}\varepsilon_{t}^{R O G R^{+}} \\ \varepsilon_{t}^{R O G R^{-}} \\ \varepsilon_{t}^{R E R} \\ \varepsilon_{t}^{I N F} \\ \varepsilon_{t}^{R N G D P}\end{array}\right]=\left[\begin{array}{ccccc}X & 0 & 0 & 0 & 0 \\ 0 & X & 0 & 0 & 0 \\ 0 & 0 & X & 0 & 0 \\ 0 & 0 & 0 & X & 0 \\ 0 & 0 & 0 & 0 & X\end{array}\right]\left[\begin{array}{c}u_{t}^{R O G R^{+}} \\ u_{t}^{R O G R^{-}} \\ u_{t}^{R E R} \\ u_{t}^{I N F} \\ u_{t}^{R E G D P}\end{array}\right]$

\section{B.2. NARDL model}

The asymmetric autoregressive distributed lag (NARDL) model of Shin et al. (2013) applied to the paper context is given by,

$\triangle L R G D P_{t}=\rho Z_{t-1}+\sum_{j=1}^{p-1} \gamma_{j} \Delta L R G D P_{t-j}+\sum_{i=1}^{q-1}\left(\theta_{i}^{+} \Delta L R O G R_{t-i}^{+}\right.$

$\left.+\theta_{i}^{-} \Delta L R O G R_{t-i}^{-}\right)+\sum_{i=1}^{q-1} \delta_{i} \Delta L R E R_{t-i}+\sum_{i=1}^{q-1} \pi_{i} \Delta I N F_{t-i}+\varepsilon_{t}$

Where,

$Z_{t-1}=L R G D P_{t-1}-\beta^{+} L R O G R_{t-1}^{+}-\beta^{-} L R O G R_{t-1}^{-}-\omega L R E R_{t-1}-\tau I N F_{t-1}$

$\mathrm{i} \phi_{j}$ is the autoregressive parameters of the model. $\theta_{i}^{+}$and $\theta_{i}^{-}$are the asymmetric distributed-lag parameters. $\varepsilon_{\mathrm{t}}$ is the error term which is supposed to be an i.i.d process with zero mean and constant variance.

The positive and negative asymmetric adjustment behavior from the initial equilibrium (before the shock) to the new equilibrium (after the shock) are given respectively by

$\boldsymbol{m}_{h}^{+}=\sum_{j=0}^{h} \frac{\partial L R G D P_{t+j}}{\partial L R O P_{t}^{+}}, \quad \boldsymbol{m}_{h}^{-}=\sum_{j=0}^{h} \frac{\partial L R G D P_{t+j}}{\partial L R O P_{t}^{-}}, h=0,1,2$,

\section{Results}

Our main results can be summarized as follows:

1. AB-SVARX results (Short-run)

The responses of non-oil real GDP to negative shocks on real oil and gas revenues are very limited and die completely after one quarter.

Resilience of the Qatar economy to negative oil and Gas revenues shock
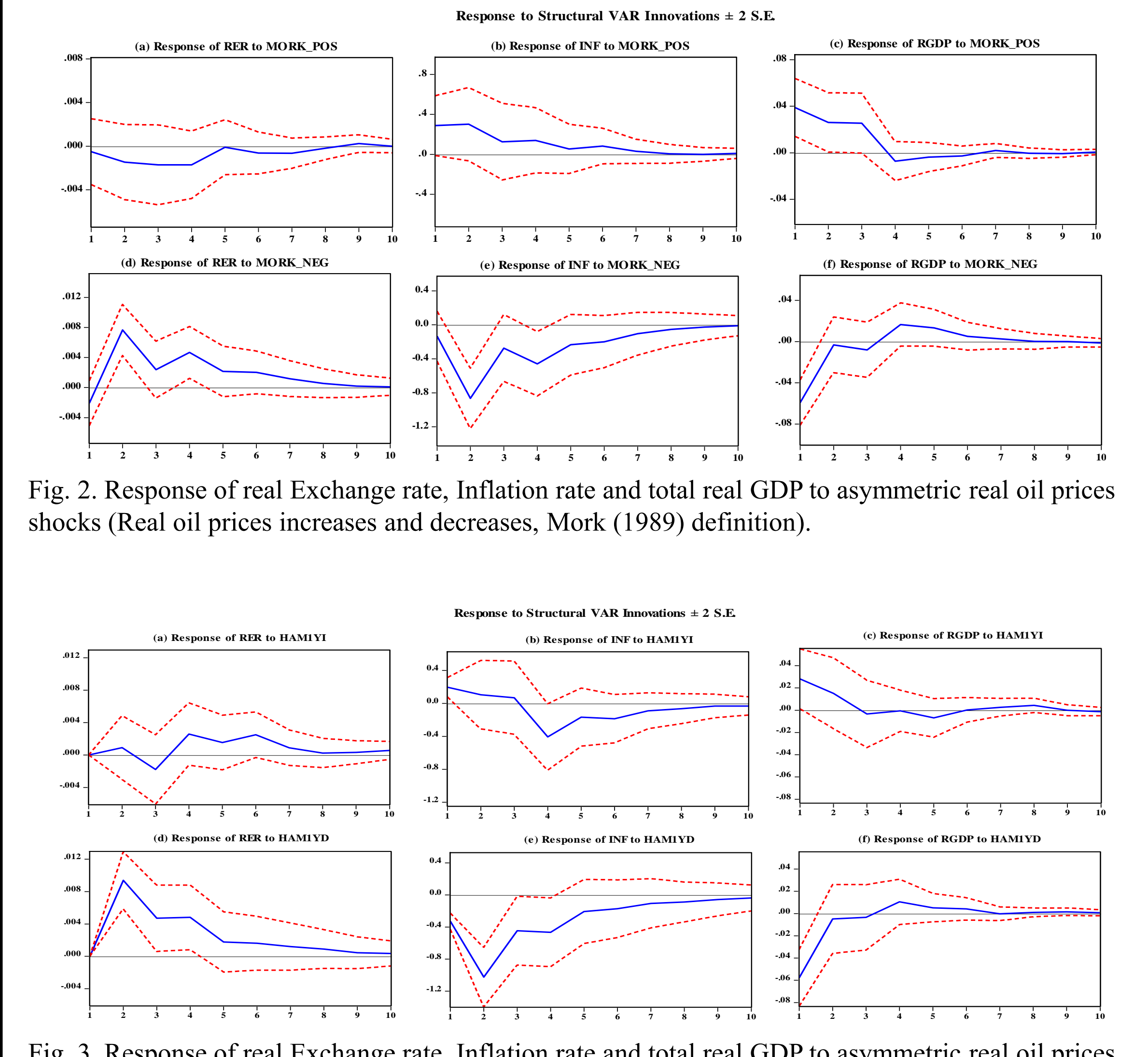

Fig. 3. Response of real Exchange rate, Inflation rate and total real GDP to
shocks (Net oil prices increases and decreases, Hamilton (1996) definition)
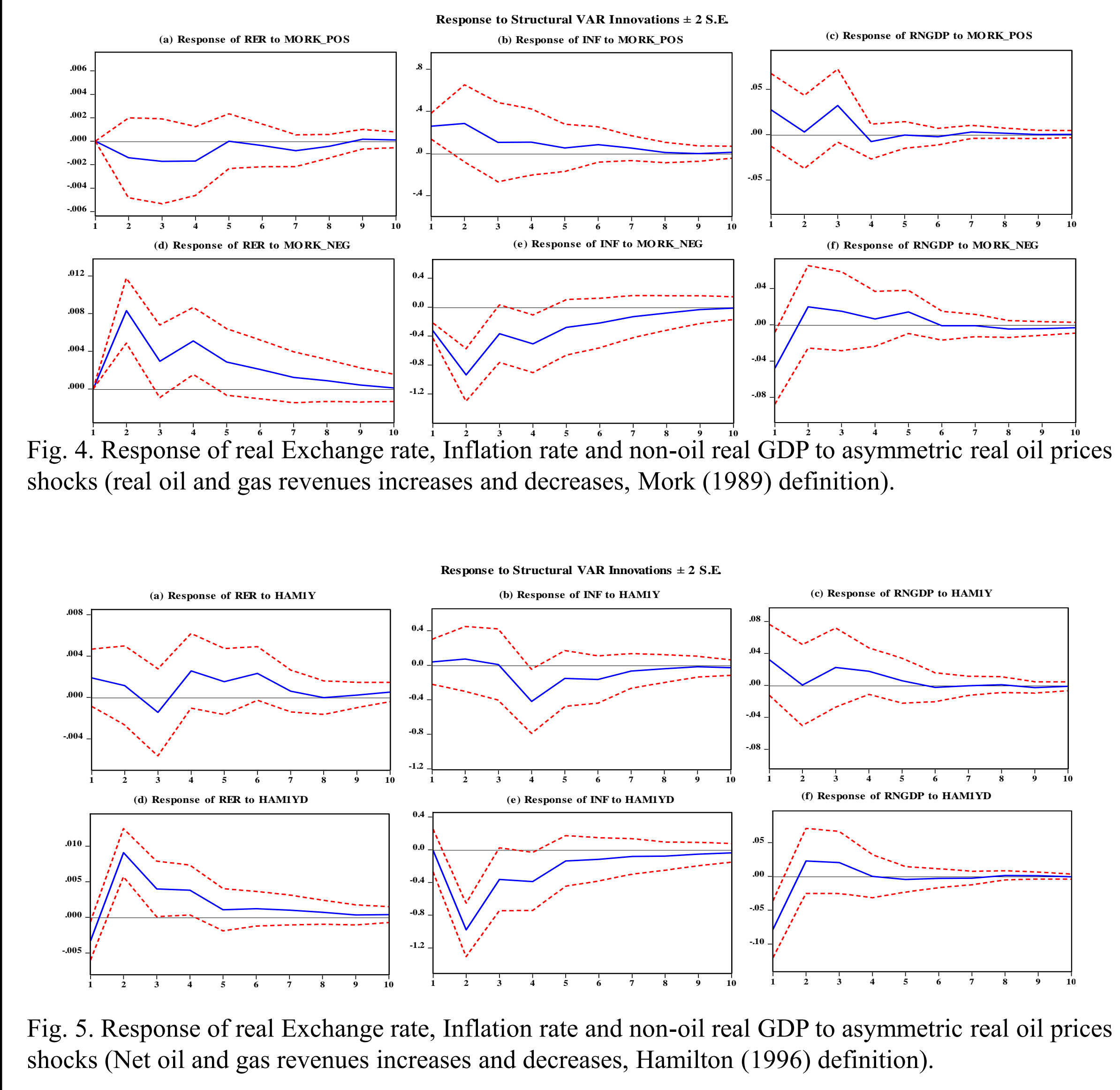

2. NARDL model results (Short- and Long-run)

$\square \underline{\text { Short-run }}$

- Real oil and gas revenue increases have a significant impact on the Qatar economic diversification. (Table 1)

- Oil and gas revenues decreases does not have a significant on non-oil economic activity. (Table 1)

Long-run: Only positive oil and gas revenue changes have a significant impact on non-oil real GDP ( $1 \%$ increase in real oil and gas revenues raises the non-oil real GDP by $0.71 \%$ ). (Table 1 )

$\Rightarrow$ The insignificant effect of oil and gas revenues decrease on the non-oil real GDP indicate the resilience of the Qatar economic to period of oil price decreases.

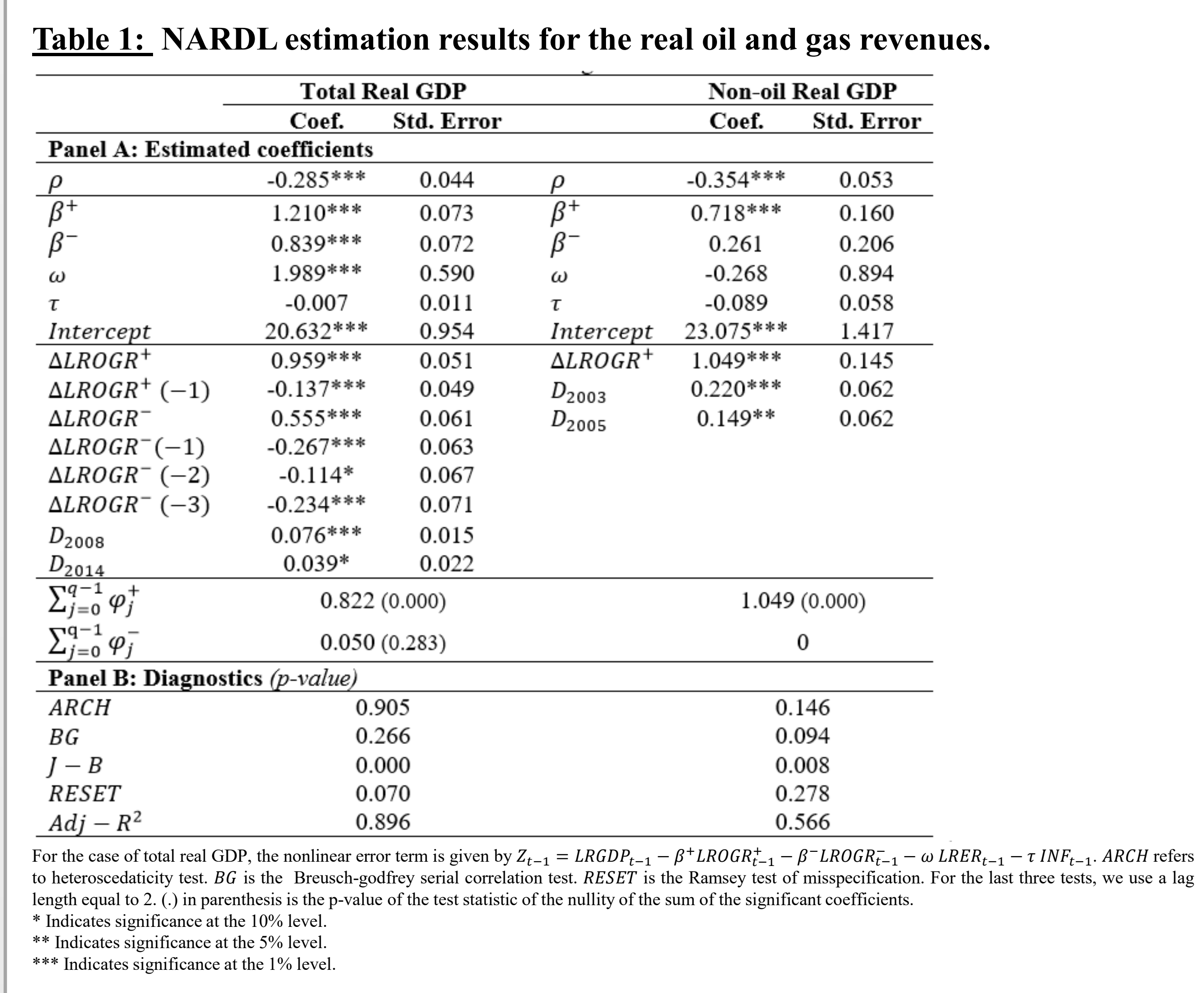

\section{Policy Implications}

The implications and proposed recommendations of our empirical findings are:

Pursuing the process of economic diversification and increasing it speed mainly in periods of rising oil and gas revenues (because of the positive impact of O\&G revenues on RNGDP).

Moving from a pegged local currency with the US. dollar to a more flexible Currency regime (the monetary authorities will re-gain the monetary policy).

The resilience of the Qatar economy offers a great opportunity to Qatari policymakers to promote the development of some specific non-oil sectors that are characterized by their higher comparative advantages.

Attracting foreign investors and encourage the creation of local smal and medium-sized enterprises to support the development of nonhydrocarbon activities.

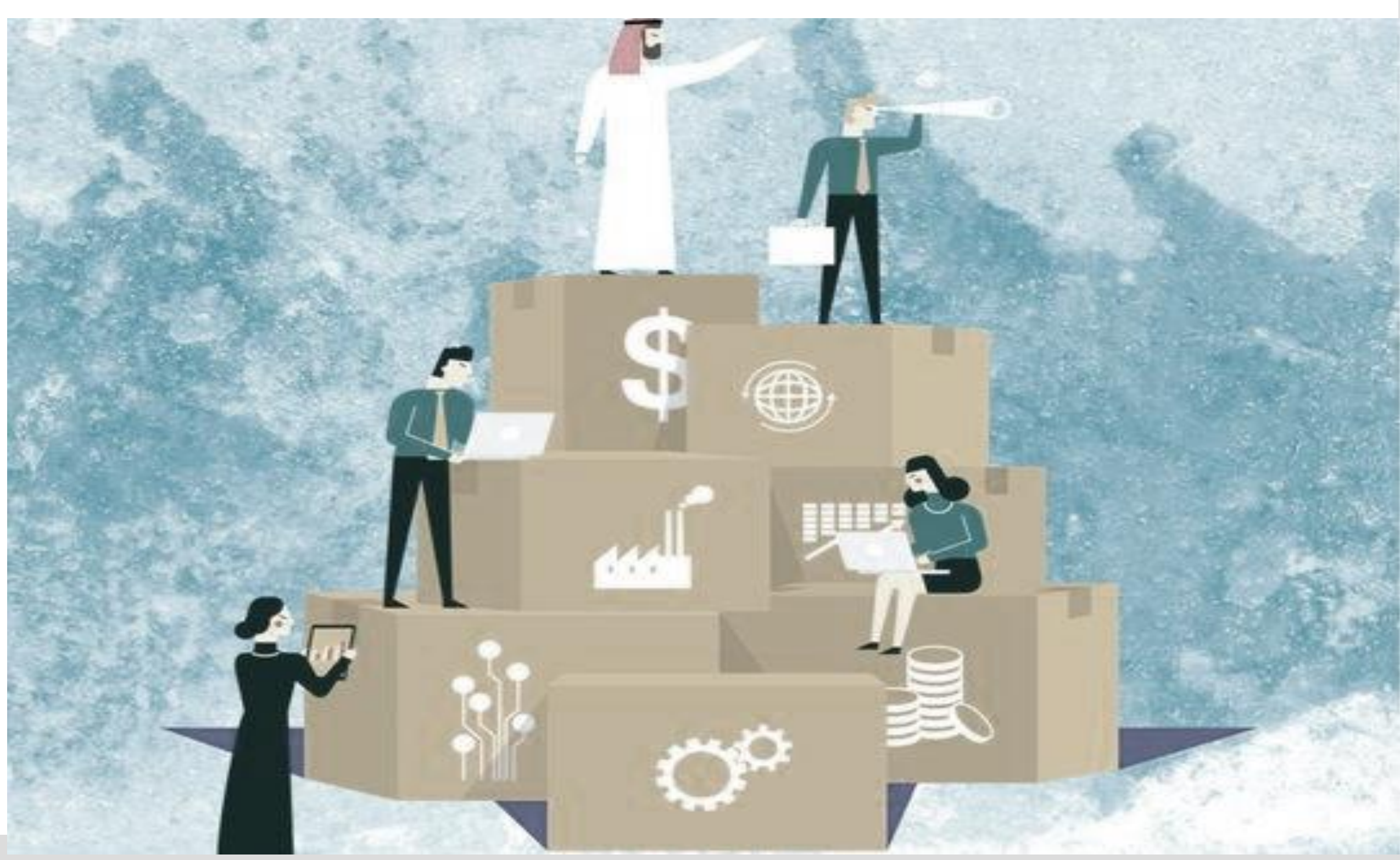

\section{Conclusions}

Our study shows that real oil and gas revenues increases have a positive and highly significant impact on the non-oil sector, which indicate the success at re-investing the oil and gas revenues for promoting and developing the non-oil sectors. Furthermore, the insignificance of the coefficient associated with the real oil price decreases indicates the resilience of the Qatar economy and the success of Qatari policymakers in diversifying their economy.

\section{How to find the paper?}

Charfeddine, L., \& Barkat, K. (2020). Short-and long-run asymmetric effect of oil prices and oil and gas revenues on the real GDP and economic diversification in oil-dependent economy. Energy Economics, 104680. (Q1, WoS. ISI-IF=4.15, Scopus, $A^{*}$ in ABDC and 3 in JGR)

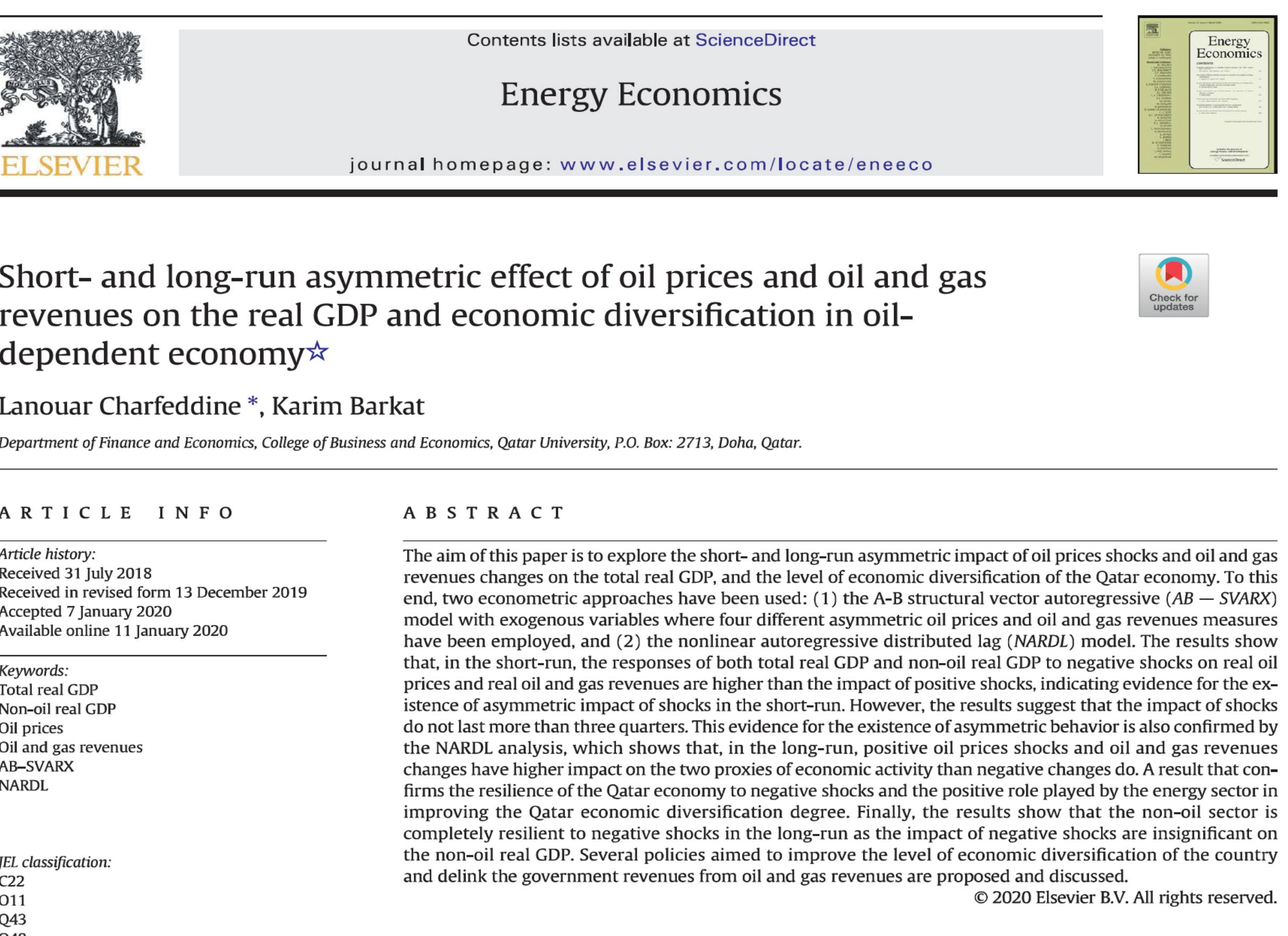

